\title{
Internal Medicine and Molecular Biology*
}

\section{Introduction}

Molecular biology, founded in the 1940s, has had a profound influence on the field of medicine in recent years. The development of genetic engineering technology, especially the discoveries of DNA modifying enzymes, methods for directing recombination of DNA molecules, and rapid methods for determining DNA and RNA nucleotide sequences, have brought us to a new frontier in the study of human genes. A direct relationship between molecular biology and internal medicine is now recognized. The introduction and application of molecular biology from bench to bedside promises solutions to previously unanswerable problems of clinical disorders.

Here, the present and future strategies for the application of molecular biology in diagnosis, etiopathogenesis and treatment are discussed with particular reference to connective tissue diseases and autoimmune disorders.

\section{Diagnosis and Molecular Biology}

The techniques of molecular biology have had an enormous impact on our understanding of the genetic basis of diseases, prenatal diagnosis and localization of genes associated with specific diseases. One of the first groups of diseases to be diagnosed using recombinant DNA technology was the hemoglobinopathies. The technique, based on the principle of restriction fragment length polymorphism (RFLP), can be used to determine linkage with diseases and anonymous polymorphic genes. More than 100 genetic diseases can now be diagnosed through the use of cDNA probes. Another new strategy involves the use of polymerase chain reaction (PCR). This technique makes it possible to amplify a specific DNA sequence more than a million fold. Thus, diagnosis can be carried out on extremely small samples and with a level of sensitivity that would have been unimaginable even a few years ago.

\section{Pathogenesis and Molecular Biology}

1) New methods for the detection of autoantibodies and their clinical significance

Autoantibodies against various cellular constituents characterize the connective tissue diseases, and have close associations with specific clinical manifestations. A recently developed immunoprecipitation technique using radiolabeled-cell extracts makes it possible to detect a broad range of autoantibodies to RNA-associated cellular antigens with a very high level of sensitivity and specificity. Through the use of this method, many new autoantibodies have recently been identified.

2) Structure and function of autoantigens

The structure and function of a large number of cellular components have been clarified through the use of human autoantibodies as probes. Moreover, a number of new cell structures have been demonstrated for the first time through the use of these antibodies. It has been learned that most autoantigenic proteins are joined with either DNA or RNA, and are involved in replication, transcrip-

*Presidential Address Presented at the 87th Annual Meeting of the Japanese Society of Internal Medicine, April 5, 1990 in Tokyo 
tion, splicing or translation of genes.

3) Mechanism of autoantibody production and autoantigen structure

Autoantibody specificity can be related to the structure of autoantigens. The U1 snRNP is a target of both anti-U1RNP and anti-Sm antibodies, and contains both epitope proteins recognized by antiU1RNP (68K, A and C) and anti-Sm (B'/B and D) autoantibodies.

We have cloned a cDNA encoding the B polypeptide of U1 snRNP particle and analyzed the antigenic epitopes. These studies demonstrate that there are at least three independent epitopes on the B polypeptide. Of these, one is present repeatedly and has homology with an epitope found in the $\mathrm{A}$ and $\mathrm{C}$ polypeptides. This finding suggests that a repeated sequence in a molecule or a sequence within a self molecule sharing homology with an exogenous structure could be recognized as an autoepitope.

4) Molecular cloning of autoantigens and autoepitopes

We have also succeeded in cloning and sequencing a cDNA encoding the autoantigen $\mathrm{Ku}$. This cDNA was expressed as a fusion protein and the latter was used as an antigen substrate in ELISA for the detection of anti-Ku antibodies. This assay system was shown to be sensitive and specific for anti- $\mathrm{Ku}$ antibodies. This system enables us to identify epitope-specific autoantibodies and the corresponding amino acid sequences. We demonstrated that the reactivity to each epitope of the $\mathrm{Ku}$ protein differs among patients and appears to be associated with individual clinical manifestations.

\section{Etiology and Molecular Biology}

1) HLA and other disease susceptibility genes

Connective tissue diseases are thought to be polygenic disorders. The first disease susceptibility gene associated with connective tissue diseases was HLA. HLA typing is now done using cDNA probes. In addition, rapid progress is now being made in the identification of the specific amino acid sequences associated with individual alleles of HLA genes. More recently, specific genes for $\mathrm{T}$ cell receptors and immunoglobulin variable regions have been shown to be associated with connective tissue diseases.

2) Gene polymorphism of autoantigen: an etiology?

It is presently thought that changes in structure and/or expression of oncogenes plays an essential role in the development of a wide variety of malignancies. In a similar manner, We have investigated whether genes encoding autoantigens have an abnormal structure or expression in autoimmune disease patients.

They performed Southern blot analyses for genomic DNA from normal subjects and autoimmune patients using various cDNA encoding autoantigens. Among the cDNAs, only the cDNA encoding the $80 \mathrm{kDa}-\mathrm{Ku}$ protein hybridized with a polymorphic DNA band when genomic DNA was treated with restriction enzyme Hind III. Moreover, the presence of the polymorphic $2.8 \mathrm{~kb}$-Hind III fragment appeared to be associated with patients with SLE who had anti-U1RNP antibodies, suggesting a possible linkage between autoantigen gene(s) and certain disease susceptibility gene(s).

(3) Virus and autoimmune diseases

In recent years, an interaction between autoantigens and viruses has been proposed. Autoantigen La/ SSB protein is a transcription terminating factor of RNA polymerase (pol) III and is found to be associated with all new RNA pol III transcripts. These RNAs include not only various host RNAs but also small RNAs of viral origin. Aminoacyl-tRNA synthetases, recognized by autoantibodies in polymyositis patients, bind genomic RNAs of a picornaviruses and catalyze aminoacylation of these viral RNAs. Thus, it can be proposed that viral infection sets in motion some process within a cell that leads to a breakdown of immunological tolerance and induction of a trigger mechanism for autoantibody production. 
4) Molecular mimicry theory

Numerous cDNAs which encode major autoantigens have been cloned and sequenced. These studies have demonstrated that a number of autoantigens contain amino acid sequences which are homologous to certain viral proteins.

A homologous amino acid sequence was first reported between the $68 \mathrm{~K}$ protein component of U1RNP and the retroviral protein $\mathrm{p} 30^{\mathrm{gag}}$. Such molecular mimicry has also been recognized between DNA topoisomerase I and $\mathrm{p} 30^{\mathrm{gag}}, 70 \mathrm{kDa}-\mathrm{Ku}$ protein and v-myc, and aminoacyl-tRNA synthetases and EB virus or influenza virus.

These findings strongly suggest the possibility of viral infection in autoimmune diseases as an etiologic factor.

\section{Treatment and Molecular Biology}

1) Clinical use of recombinant bioreactive peptides

Many valuable polypeptides have been developed by recombinant DNA techniques and have been put into practical use. These include hormones (insulin, human growth hormone, erythropoietin and G-CSF), cytokines (interferons, interleukin-2), and vaccines (HB vaccine, etc). These materials are produced in large quantities and in standardized qualities, so that stable therapeutic effects can be expected.

In the future, protein engineering techniques will make possible the alterations of selected amino acid sequences of natural polypeptides to make new bioactive molecules with stronger therapeutic effects and fewer complications.

\section{2) Gene therapy}

The ultimate goal for treatment of genetic diseases and gene-involving disorders is gene therapy. At present, there are still many technical problems and ethical dilemmas to be resolved before clinical application.

\section{Conclusions}

The technology of molecular biology has been indispensable in the progress of internal medicine. However, the contribution in these two fields has not been a one-way relationship, because the findings in internal medicine have also contributed to the development of molecular biology. For example, autoantibodies found in patients with autoimmune diseases have been utilized to elucidate the structure and function of unknown cellular molecules and they have served as useful probes for molecular cloning. It can be expected that internal medicine and molecular biology will continue to develop hand-in-hand. It is important to keep in mind the ethical issues that are involved when we begin to apply such powerful technology to human disease and to the understanding and control of the processes which operate in human life. I believe that internal medicine should take a leadership role in the efforts to fulfill the promise for an improved quality of life which is now offered by the introduction of molecular biology.

\section{REFERENCE}

Mimori $\mathrm{T}$, et al. Isolation and characterization of cDNA encoding the $80-\mathrm{kDa}$ subunit protein of the human autoantigen $\mathrm{Ku}(\mathrm{p} 70 / \mathrm{p} 80)$ recognized by autoantibodies from patients with scleroderma-polymyositis overlap syndrome. Proc Natl Acad Sci USA 87: 1777, 1990. 